\title{
Tooth-colored Restoration in Molars: Three Case Reports
}

\author{
${ }^{1}$ Prachi Mital, ${ }^{2}$ Charu Thanvi, ${ }^{3}$ Deepak Raisingani, ${ }^{4}$ Ashwini B Prasad, ${ }^{5}$ Amit Bhamboo, ${ }^{6}$ Harshit Srivastava
}

\begin{abstract}
As technology progresses, the basic desires and expectations of humans are also increasing day by day. Esthetics is becoming the prime concern of patients even more than treatment. Here comes the role of esthetic restorations for anterior teeth as well as posterior teeth. An inlay or onlay is a more conservative and long-lasting way of restoring the tooth than metal filling, composite, and even a crown. Inlays are intracoronal restoration which may cap one or more, but not all of the cusps, and onlays are also a type of intracoronal restoration which cap all of the cusps of tooth. Most indirect restorations are made on a replica of the prepared tooth in a dental laboratory by a trained technician. Ceramic inlays and onlays have become popular not only because of patient demand for esthetic, durable restorative materials, but also because of recent improvements in materials, fabrication techniques, and bonding systems. The traditional fillings reduce the tooth strength by up to $50 \%$ whereas inlay or onlay made up of high-strength porcelain or ceramic can actually increase tooth strength up to $75 \%$ and lasting 10 to 30 years. Therefore, maximum amount of tooth structure is conserved with the help of inlay and onlay as compared with crown. And the strength provided by tooth itself is much higher than any restorative material, the best way to maintain and improve the strength of tooth is preservation of the tooth
\end{abstract}

Keywords: Bonding, Composite, Inlay, Onlay, Resin.

How to cite this article: Mital $P$, Thanvi $C$, Raisingani $D$, Prasad AB, Bhamboo A, Srivastava H. Tooth-colored Restoration in Molars: Three Case Reports. J Mahatma Gandhi Univ Med Sci Tech 2017;2(3):176-180.

Source of support: Nil

Conflict of interest: None

\section{INTRODUCTION}

The word esthetic means concern with the beauty or the appreciation of beauty. And where esthetic is concerned, the dentist comes in mind and conservative dentistry

\footnotetext{
${ }^{1,5,6}$ Assistant Professor, ${ }^{2}$ Resident, ${ }^{3}$ Professor and Head ${ }^{4}$ Associate Professor

${ }^{1-4,6}$ Department of Conservative Dentistry and Endodontics Mahatma Gandhi Dental College and Hospital, Jaipur, Rajasthan India

${ }^{5}$ Department of Oral and Maxillofacial Surgery, Mahatma Gandhi Dental College and Hospital, Jaipur, Rajasthan, India

Corresponding Author: Charu Thanvi, Resident, Department of Conservative Dentistry and Endodontics, Mahatma Gandhi Dental College and Hospital, Jaipur, Rajasthan, India, Phone: +919828854511, e-mail: kusumvyas41164@gmail.com
}

gained popularity in the world of beauty. Recreating the original anatomy of the tooth resembling the natural appearance is a big challenge for the dentist. Good personalized smile comprises shade, shape, and surface texture of material. Studies are going on since the $1980 \mathrm{~s}$ in search of a durable and tooth-colored restorative dental material, which strengthens the tooth with great esthetic appearance. Various materials used are composite resins, glass ionomer cement, all-ceramic, and ceramic bonded to metal. Since the esthetic is in high demand, the dentist is also concerned about the tooth preparation, application, cementation, advantages, and limitations of specific material to achieve the most desirable results. Also the success rate of restoration in posterior teeth depends on case selection, the preparation, design, ceramic materials, and, most importantly, the creation of ceramic/material interface. ${ }^{1,2}$

Inlays and onlays are types of indirect restorations used when the tooth is damaged too much to support the basic direct fillings but not so severe that it needs crown. ${ }^{3}$ Inlay /onlays are prepared outside the patient's mouth, in the laboratory, and then are cemented or bonded to the tooth. These restorations fit the reduced tooth in a lock-and-key manner and are strong enough to rebuild the damaged tooth structure. These restorations constitute an elective alternative to amalgam restorations and other tooth-colored restorations. When strength is a factor, we need to select the material which can sustain the high occlusal forces, which improves the prognosis of tooth. ${ }^{4,5}$

\section{CASE REPORTS}

\section{Case 1A}

A 28-year-old male patient reported to the Department of Conservative Dentistry and Endodontics with the complaint of food lodgment and sensitivity of tooth in upper posterior tooth region. The clinical examination of tooth incorporation with radiographic examination (Fig. 1) revealed a wide mesioproximal caries in upper left 1st molar, which was the reason for food lodgment to the patient; the margins of tooth were supragingivally located, and the mesiobuccal width of proximal region was significantly wide, which drew the treatment plan toward indirect restoration instead of direct for better prognosis. For the patient, esthetics was the prime 


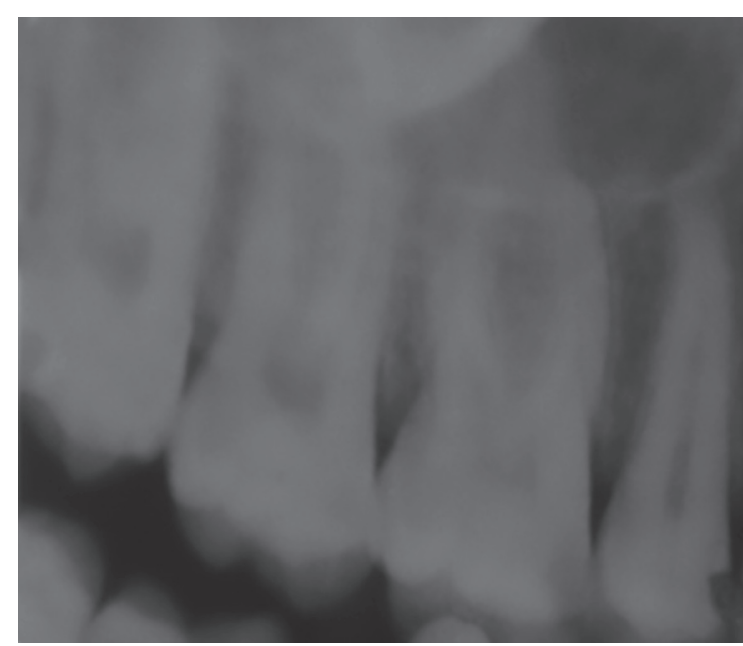

Fig. 1: Preoperative radiograph

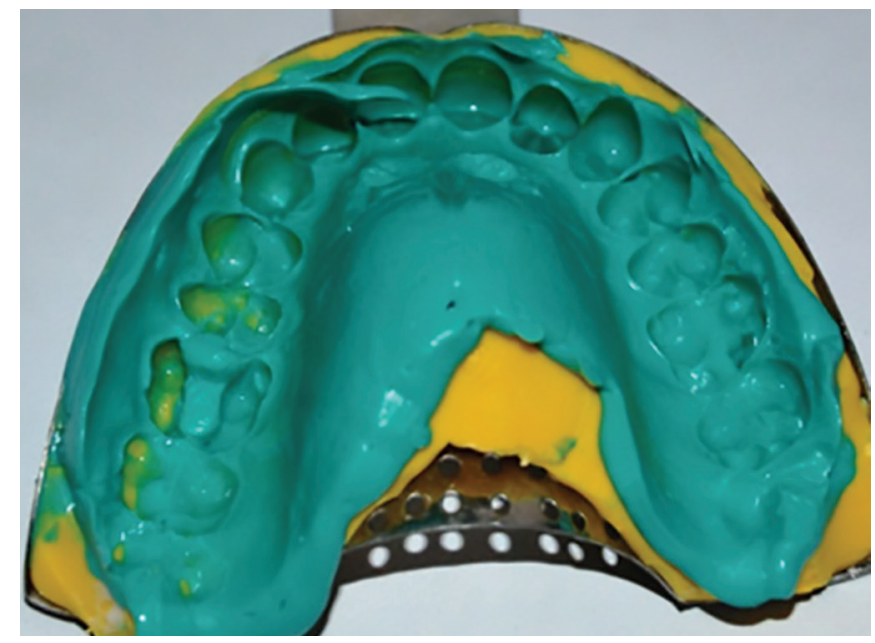

Fig. 3: Full arch impression

concern, so we planned for ceramic inlay to restore the defect with beauty similar to natural appearance. The procedure starts with marking the bite of patient with articulating paper. The cavity preparation was done with the principles of cavity preparation (Fig. 2). After cavity was prepared, the impressions were made using a silicone material (high viscosity washed with low viscosity) (Aquasil Ultra, Dentsply, Clauk, Milford, USA) (Fig. 3) and the model was sent to lab where BruxZir inlay was made using computed-aided design and computer-aided manufacturing technique (Fig. 4).

The intraoral fit was checked, the prepared inlay restoration was etched, silenated, and cemented using Calibra resin cement (Dentsply Caulk, Milford, USA) (Fig. 5). Figure 5 shows the restoration, which is indistinguishable from the tooth being restored, after cementation. Also the patient was satisfied form esthetic inlay restoration of damaged tooth. After a 12-month followup, the radiographic and clinical examination of tooth illustrated good interproximal bone level and the gingi-

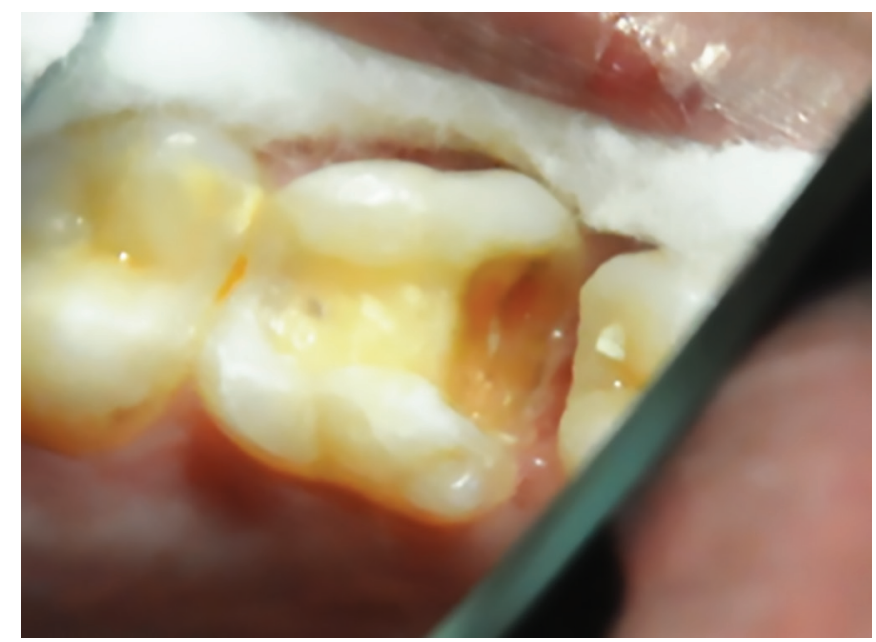

Fig. 2: Cavity preparation

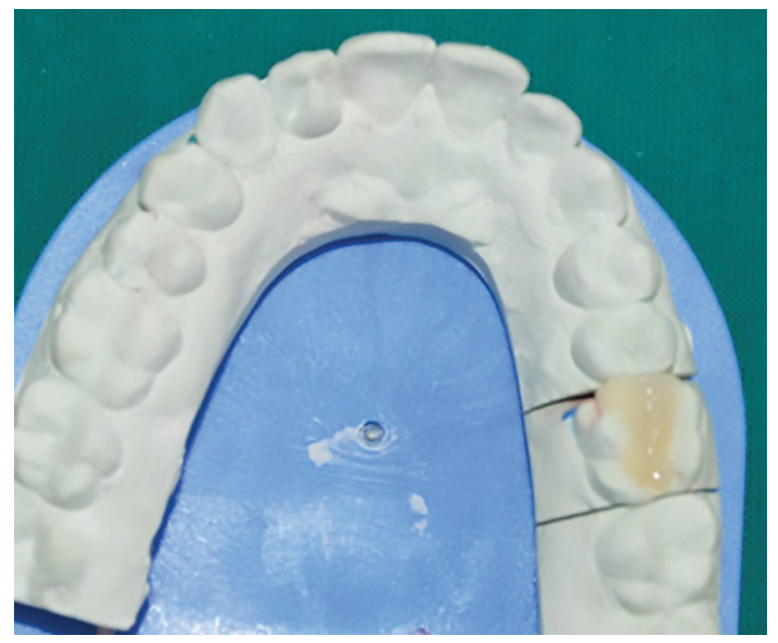

Fig. 4: Inlay on die

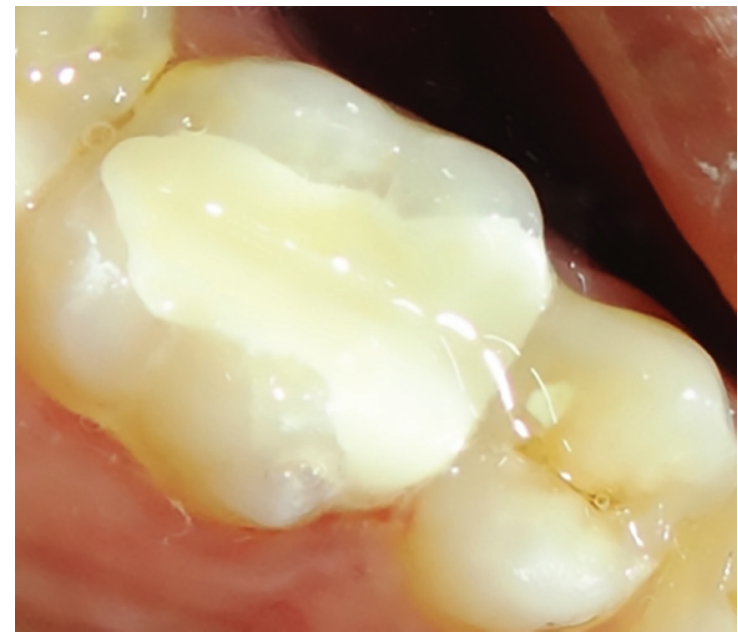

Fig. 5: Cementation of inlay

val tissues were perfectly healthy; clinically, there was no discoloration of restoration, chipping of material, and the occlusion was in complete harmony with no complain of food lodgment or dislocation of restoration (Fig. 6). 

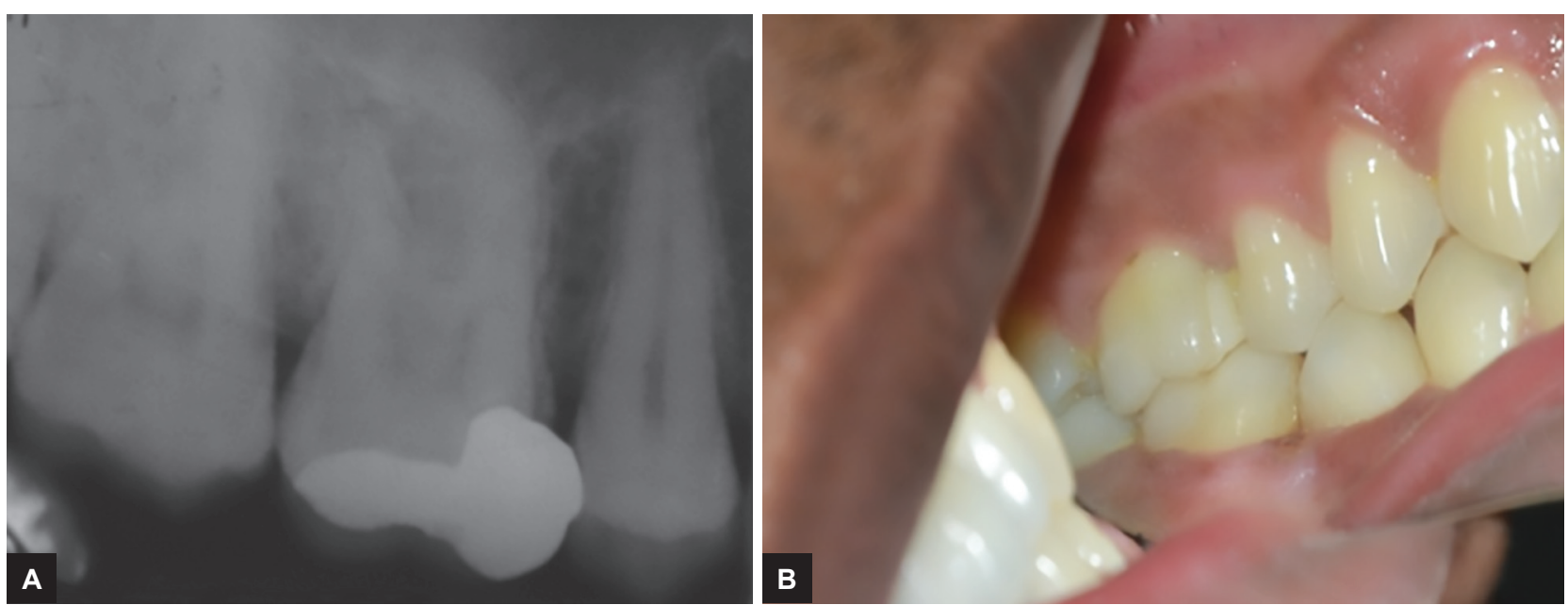

Figs 6A and B: (A) After 12 months follow-up. (B) After 12 months follow-up clinical view

\section{Case 1B}

In another case in which the patient came to our department with the complaint of fractured filling, the clinical and radiographic evidence showed a fractured restoration with caries in the proximal portion of tooth (Fig. 7). On excavating, all the caries of the tooth became an absolute indication for indirect restoration, as the decay involved a large area of tooth which might get fractured if direct restoration was attempted. The cavity was designed, impression of the arch was taken, die was prepared and sent to lab where BruxZir inlay was made and then the inlay restoration was etched, silenated, and cemented on the concerned tooth in the same way as done in case 1A. Figure 8 shows the clinical photograph of the patient after the cementation of ceramic inlay, showing proper occlusal anatomy and marginal fit with phenomenal proximal contact and contour with no overhangs, which will help the gingiva to remain healthy. The damage was replaced with an esthetically healthy restoration, which rejuvenated the tooth with all its forms and function.

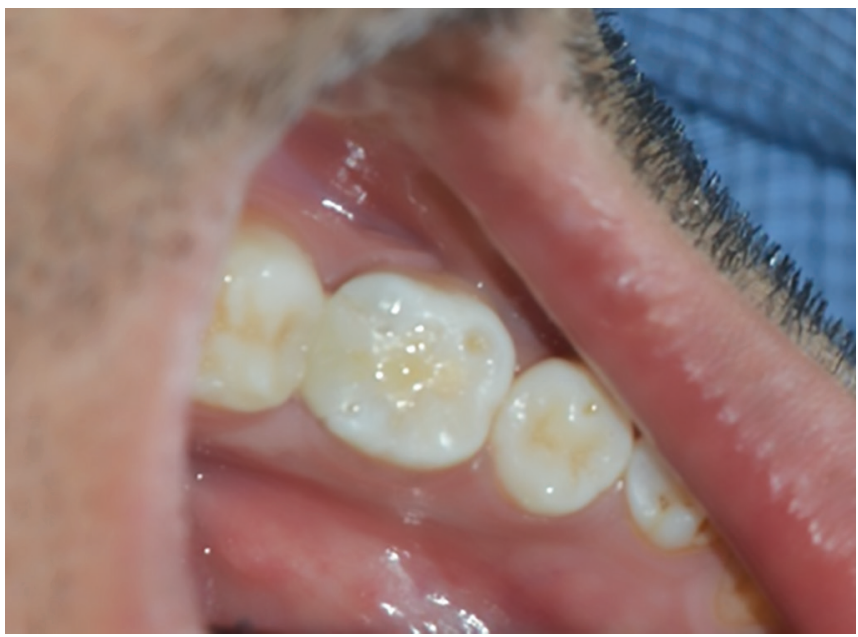

Fig. 8: Postoperative view

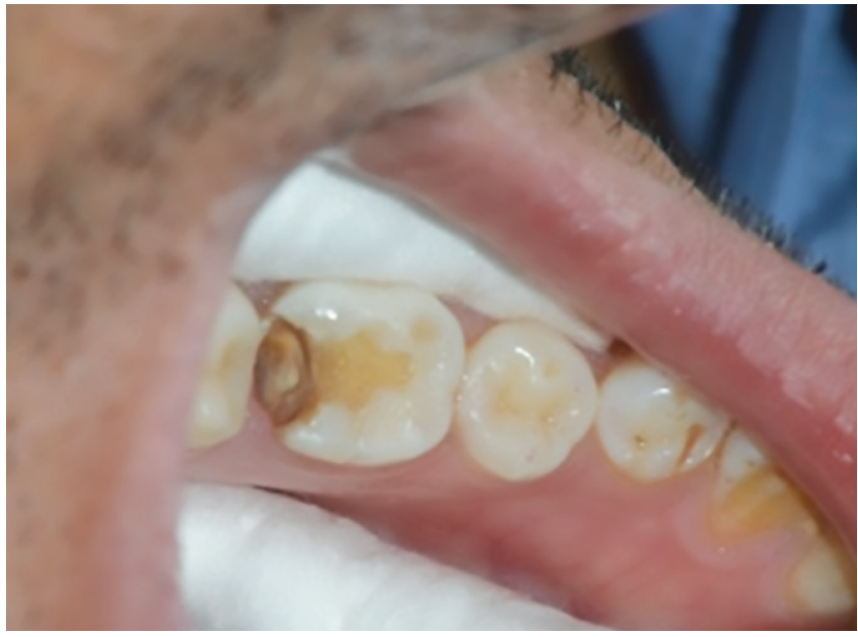

Fig. 7: Preoperative view

\section{Case 2}

A 26-year-old male patient came to the department with a complaint of pain in lower left back tooth region, and the radiographic and clinical examination indicated the tooth for root canal treatment (RCT). The RCT was performed in concerned tooth (Fig. 9) with a conservative

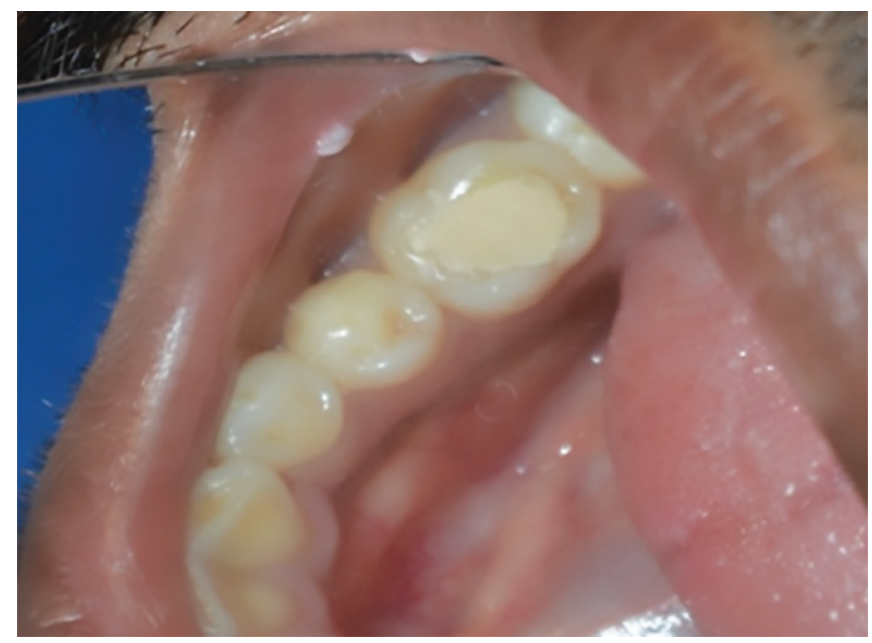

Fig. 9: After RCT 


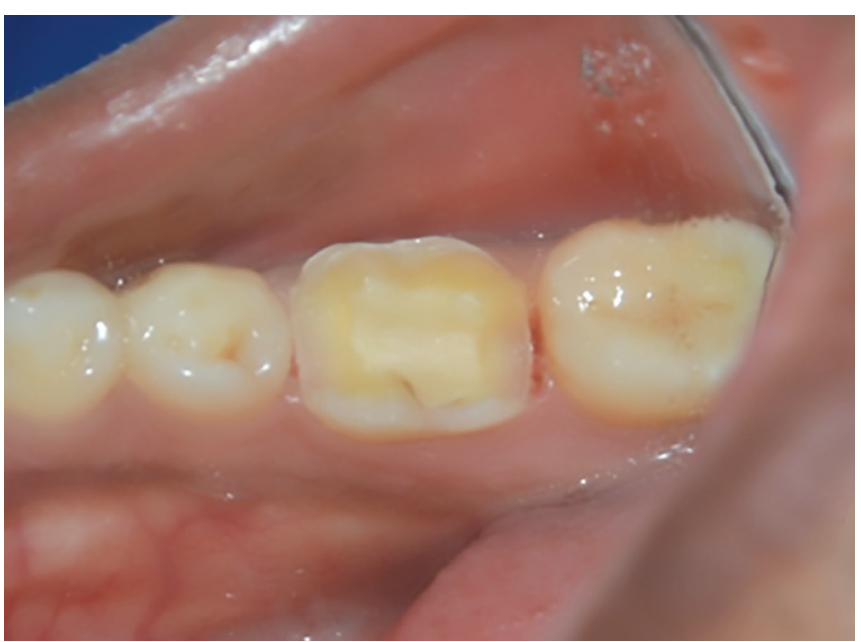

Fig. 10: Cavity preparation

access cavity preparation, and since the posterior teeth received greater stress, an onlay restoration was planned instead of full crown with the thought of preservation of tooth structure, strength, and retention as well. No. 271 carbide bur was used in cavity preparation following the principles of cavity preparations (Fig. 10). The retraction cord was tucked in gingival sulcus and full arch impression was taken using a silicone material (high viscosity washed with the low viscosity) followed by master cast and die preparation of concerned tooth with the ceramic onlay try-in over the die (Fig. 11). The onlay was placed on tooth and the occlusal fit was checked, followed by cementation with the help of resin cement (Fig. 12). Figure 13 illustrates the clinical and radiographic view of tooth. On a 7-month follow-up, it shows healthy gingival contour, good interdental bone level, proper occlusion, and proximal contact with no discoloration of crown.

\section{DISCUSSION}

The primary emphasis for dentistry now is the preservation of tooth structure ${ }^{6}$ and reinforcement of tooth by

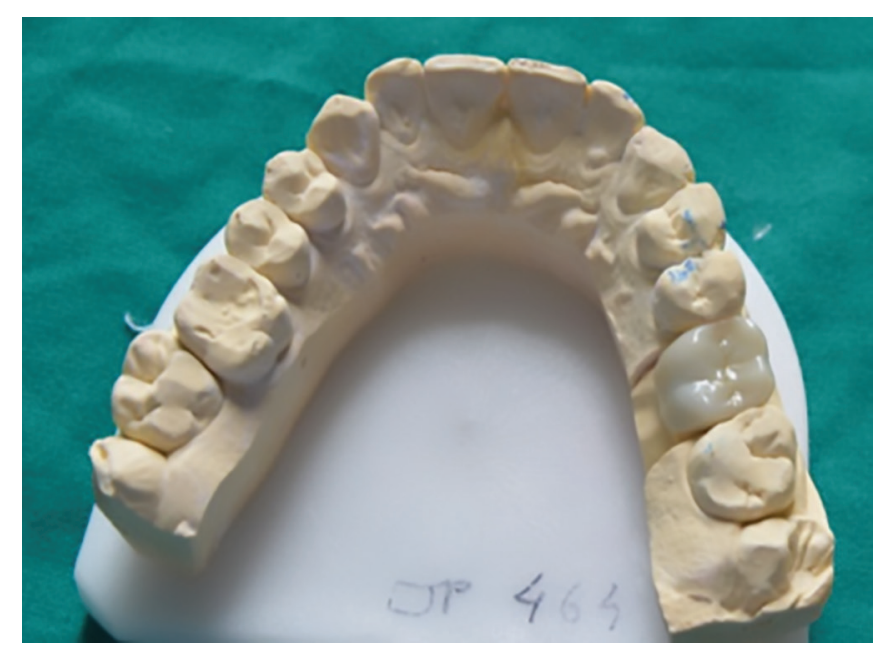

Fig. 11: Onlay on die

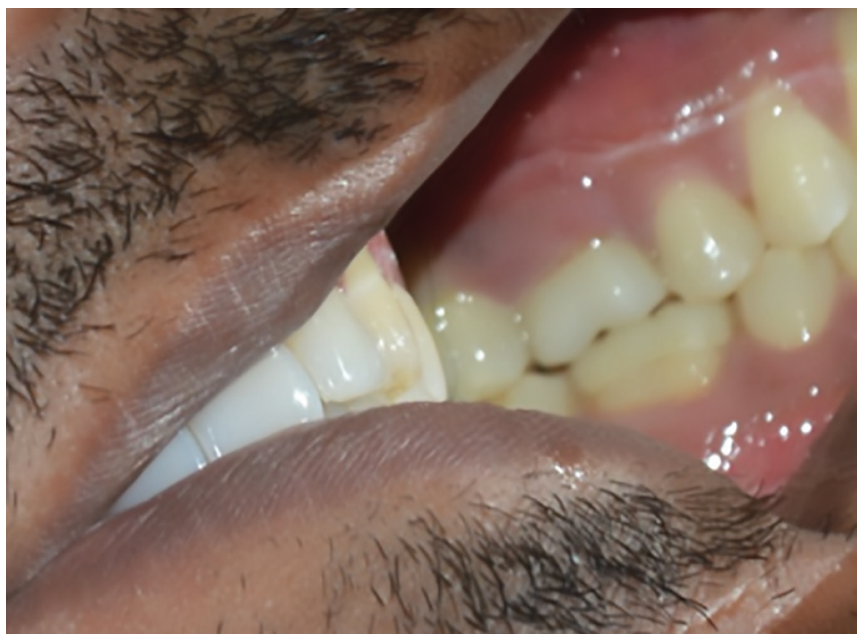

Fig. 12: Cementation

reducing the fracture incidence of restored tooth. ${ }^{7,8}$ In fact, preserving sound tissue by using a minimally invasive bonded restoration resulted in less trauma and superior prognosis. ${ }^{8}$ These are the prime reasons which have brought the ceramic inlays and onlays in great demand
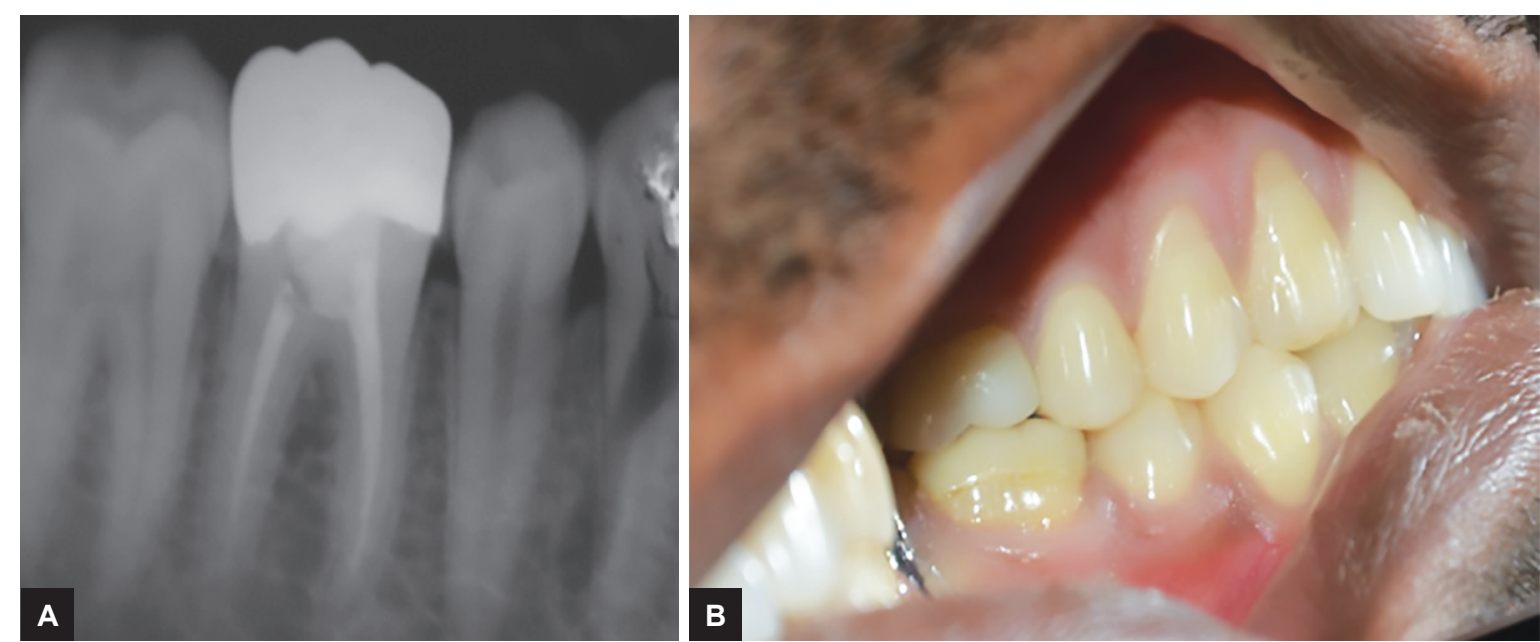

Figs 13A and B: (A) A 7 months follow-up radiographic view. (B) A 7 months follow-up clinical view 
and acceptance. The excessive wide cavities may preclude the use of direct posterior composite restorations and here, the indirect restorations came into action for restoration of tooth, as they found it superior over direct restorations in numerous ways, such as high biocompatibility, they do not encourage the concentration of dental plaque in their surfaces with perfect color stability, and also ceramic is stronger than composite resins and offers superior physical properties. BruxZir ceramic inlays and onlays offer mechanical advantages like high flexure strength $(12,000 \mathrm{MPa})$, high fracture toughness, and excellent resistance to thermal shock, as compared with direct resin, titanium, amalgam, and gold restorations for treating vital posterior teeth. They also provide esthetic restorations with a translucent natural aspect, which is indistinguishable from the tooth being restored. In the same context, they conserve tooth structure and offer mechanical benefits due to modern adhesive technology. ${ }^{2}$ In addition, the bonding of inlays-onlays is done with Calibra resin cement, which is a simple solution that has superior clinical performance, with no need for selfcure activator and no mixing of primers, and it increases the fracture resistance caused by large mesial-occlusaldistal preparations. ${ }^{6}$ Also, to retain metal fillings, more tooth structure is reduced which eventually reduces the strength of tooth.

However, the direct restorations have their own limitations regarding the extent of damage, esthetic zone, strength, etc. Direct composite restorations are not helpful in stress concentration areas, and also, the discoloration of restorations remains inevitable. The metal fillings have proved to be strong in stress-bearing areas, but require large amount of tooth structure reduction, which eventually reduces the strength of tooth. However, the indication of indirect restorations should consider many factors. They include supragingivally located margins and sufficient enamel thickness.
A successful minimally invasive approach using inlays and onlays requires a case selection and assessment. The clinician should then evaluate the tooth being treated. The margins should be supragingivally located for a secure bonding. The cavities should not be extended below the cementoenamel junction. The preparation design reduces the physical stress, leading to a strong restoration. The preparation should have smooth flowing margins to facilitate the fabrication of the restoration. Inlays and onlays offer excellent restorations that may be underused in dentistry. The technique requires multiple patient visits and excellent laboratory support, but the resulting restorations are durable and long-lasting.

\section{REFERENCES}

1. Abraham S, Attur K, Singh SK, Lisa Chacko N. Aesthetic inlays. Int J Dent Clin 2011 Jul-Sep;3(3):62-64.

2. Fligor J. Preparation design and considerations for direct posterior composite inlay/onlay restoration. Pract Proced Aesthet Dent 2008 Aug;20(7):413-419.

3. DaouahiN,HadyaouiD,NouiraZ,SaafiJ,HarzallahH,CherifM. Restoration of a compromised vital posterior tooth using ceramic inlay-onlay. J Dent Oral Care 2015 Apr;1(2):1-3.

4. Krämer N, Frankenberger R. Clinical performance of bonded leucite-reinforced glass ceramic ilays and onlays after eight years. Dent Mater 2005 Apr;21(3):262-271.

5. Felden A, Schmalz G, Federlin M, Hiller KA. Retrospective clinical investigation and survival analysis on ceramic inlays and partial ceramic crowns: results up to 7 years. Clin Oral Invest 1998 Dec;2(4):161-167.

6. Thompson MC, Thompson KM, Swain M. The all-ceramic, inlay supported fixed partial denture. Part 1. Ceramic inlay preparation design: a literature review. Aust Dent J 2010 Jun;55(2):120-127.

7. Saridag S, Sevimay M, Pekkan G. Fracture resistance of teeth restored with all-ceramic inlays and onlays: an in vitro study. Oper Dent 2013 Nov-Dec;38(6):626-634

8. Denry IL, Rosenstiel SF. Flexural strength and fracture toughness of Dicor glass-ceramic after embedment modification. J Dent Res 1993 Mar;72(3):572-576. 